\title{
Letter \\ Ultrasound has the potential to detect degeneration of articular cartilage clinically, even if the information is obtained from an indirect measurement of intrinsic physical characteristics
}

\author{
Hiroshi Kuroki ${ }^{1}$, Yasuaki Nakagawa ${ }^{2}$, Koji Mori ${ }^{3}$, Masahiko Kobayashi ${ }^{4}$, Ko Yasura ${ }^{4}$, \\ Yukihiro Okamoto ${ }^{4}$, Takashi Suzuki ${ }^{4}$, Kohei Nishitani ${ }^{4}$ and Takashi Nakamura ${ }^{4}$
}

\author{
1Division of Motor Function Analysis, Department of Physical Therapy, Human Health Sciences, Graduate School of Medicine, Kyoto University, Kyoto, \\ 606-8507, Japan \\ ${ }^{2}$ Department of Orthopaedic Surgery, National Hospital Organization Kyoto Medical Center, Kyoto, 612-8555, Japan \\ ${ }^{3}$ Department of Applied Medical Engineering Science, Graduate School of Medicine, Yamaguchi University, Ube, Yamaguchi, 755-8611, Japan \\ ${ }^{4}$ Department of Orthopaedic Surgery, Graduate School of Medicine, Kyoto University, Kyoto, 606-8507, Japan
}

Corresponding author: Yasuaki Nakagawa, yanakaga@kyotolan.hosp.go.jp

Published: 24 June 2009

This article is online at http://arthritis-research.com/content/11/3/408

Arthritis Research \& Therapy 2009, 11:408 (doi:10.1186/ar2727)

(c) 2009 BioMed Central Ltd

See related research by Nakagawa et al., http://arthritis-research.com/content/10/4/R78, and related editorial by Zheng and Huang, http://arthritis-research.com/content/10/6/125

We appreciate the concern shown by Zheng and Huang [1] regarding our earlier article [2]. We presented simple data showing that the ultrasound response of articular cartilage may be related to its International Cartilage Repair Society grading, and concluded that ultrasound evaluation using the signal intensity - dependent on the ultrasound reflection coefficient at the cartilage surface - may be helpful to differentiate International Cartilage Repair Society grades, especially grade 0 from grade 1 cartilage [2].

Our ultrasound system obtains indirect information on intrinsic physical characteristics of living human articular cartilage in vivo. We recognize that the ultrasound signal intensity of articular cartilage relates to the parameters of the tissue reflection coefficient, acoustic impedance, the elastic modulus, and surface conditions. In clinical settings, however, these parameters are difficult to measure separately. We therefore consider that the signal intensity obtains information including these parameters.

We do not disregard measuring the intrinsic physical characteristics. Indeed, the signal intensity (maximum magnitude) correlated significantly with the aggregate modulus of articular cartilage [3]. We mentioned the equations of Young modulus, indicating the speed of sound, the density of a material, and the acoustic impedance of a material [4], and presented the Gabor function as the mother wavelet and equations [2]. The signal intensity did not depend on the surface curvature for radii $>40 \mathrm{~mm}$, and mainly reflects the condition from the surface of the cartilage to a depth of one wavelength (about $0.150 \mathrm{~mm}$ ) [5]. The tip of the probe with an ultrasonic transducer is designed to achieve uniform distance between the transducer and the cartilage surface.

Our ultimate goal is not to measure the intrinsic physical characteristics but to improve the diagnostic use of an arthroscopic ultrasound system and the method to detect the early stage of degeneration of human articular cartilage. The signal intensity, considering tissue histology [4] and estimation of the mechanical property of meniscus [6], was studied aiming toward human clinical study [7]. Our ultrasound system can be used with the arthroscopic probe and can obtain information on the degeneration of human articular cartilage in vivo.

It is not easy to calculate the intrinsic physical characteristics from an ultrasonic echo obtained under arthroscopy. True it is ideal that the intrinsic physical characteristics of cartilage are measured accurately, but this is still difficult in clinical settings using existing devices. The authors consider that weakness to measure the intrinsic physical characteristics accurately does not interfere with our final purpose; that is, quantitative evaluation of human cartilage in clinical settings such as arthroscopy.

Finally, information on the signal intensity is valuable for clinicians who want to know the mechanism of degeneration of cartilage without performing a tissue biopsy. Until an ultrasound system that can measure the intrinsic physical characteristics of human cartilage in vivo is developed, we believe 
that the ultrasound information our technique provides will help clinicians to understand degeneration of articular cartilage - even if the information is obtained from an indirect measurement of the intrinsic physical characteristics.

\section{Competing interests}

The authors declare that they have no competing interests.

\section{References}

1. Zheng YP, Huang YP: More intrinsic parameters should be used in assessing degeneration of articular cartilage with quantitative ultrasound. Arthritis Res Ther 2008, 10:125.

2. Kuroki H, Nakagawa $\mathrm{Y}$, Mori K, Kobayashi M, Yasura K, Okamoto Y, Suzuki T, Nishitani K, Nakamura T: Ultrasound properties of articular cartilage in the tibio-femoral joint in knee osteoarthritis: relation to clinical assessment (International Cartilage Repair Society grade). Arthritis Res Ther 2008, 10: R78.

3. Mori $K$, Hattori $K$, Habata $T$, Yamaoka $S$, Aoki $H$, Morita $Y$, Takakura Y, Tomita N, Ikeuchi K: Measurement of the mechanical properties of regenerated articular cartilage using wavelet transformation. In Tissue Engineering for Therapeutic Use 6. Edited by Ikada Y, Umakoshi Y, Hotta T. Tokyo: Elsevier; 2002:133-142.

4. Kuroki H, Nakagawa $Y$, Mori K, Ohba M, Suzuki T, Mizuno $Y$, Ando K, Takenaka M, Ikeuchi K, Nakamura T: Acoustic stiffness and change in plug cartilage over time after autologous osteochondral grafting: correlation between ultrasound signal intensity and histological score in a rabbit model. Arthritis Res Ther 2004, 6:R492-R504.

5. Mori K, Nakagawa Y, Kuroki H, Ikeuchi K, Nakashima K, Nime T, Nakamura T, Kawai S, Saito T: Non-contact evaluation for articular cartilage using ultrasound. JSME Int J Ser A 2006, 49: 242-249.

6. Yasura K, Mizuno Y, Nakagawa Y, Mori K, Takenaka M, Ohashi T, Yamada K, Kobayashi M, Ando K, Kuroki H, Suzuki T, Ikeuchi K, Tsutsumi S, Nakamura T: Estimation of the mechanical property of meniscus using ultrasound: examinations of native meniscus and effects of enzymatic digestion. J Orthop Res 2007, 25:884-893.

7. Nishitani K, Nakagawa $Y$, Gotoh T, Kobayashi M, Nakamura T: Intraoperative acoustic evaluation of living human cartilage of the elbow and knee during mosaicplasty for osteochondritis dissecans of the elbow: an in vivo study. Am J Sports Med $2008,36: 2345-2353$. 